\title{
Reflets
}

Revue ontaroise d'intervention sociale et communautaire

\section{Travail, handicap et discrimination : lorsque le travail devient aussi un espace de production du handicap}

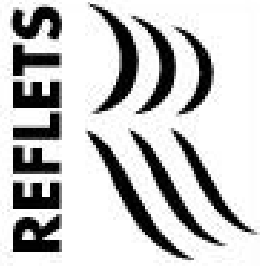

\section{Rachid Merzouk}

Volume 14, numéro 1, 2008

Travail, jeunesse et intervention

\section{URI : https://id.erudit.org/iderudit/018858ar}

Aller au sommaire du numéro

Éditeur(s)

Reflets : Revue d'intervention sociale et communautaire

ISSN

1203-4576 (imprimé)

1712-8498 (numérique)

Découvrir la revue

Citer cet article

Merzouk, R. (2008). Travail, handicap et discrimination : lorsque le travail devient aussi un espace de production du handicap. Reflets, 14(1), 155-181.

\section{Résumé de l'article}

Cet article traite de la question du lien entre travail et handicap. Ce lien est à la fois complexe et problématique. Ce qui fait du travail un champ où se jouent plusieurs processus de rejet et de discrimination. La discrimination que rencontre la personne ayant une incapacité sur le marché du travail est double : elle se produit par rapport à l'accès au travail et au sein du travail. La compréhension de cette discrimination nécessite de tenir compte d'autres formes de discrimination que la personne ayant une incapacité rencontre dans de multiples secteurs de la vie. Enfin, cette discrimination, parce que négative, appelle le renforcement des pratiques de discrimination positive en faveur de la personne ayant une incapacité, qu'elle soit déjà en emploi ou en quête d'un travail.

Tous droits réservés ( Reflets : Revue d'intervention sociale et communautaire, Ce document est protégé par la loi sur le droit d’auteur. L’utilisation des 2008 services d'Érudit (y compris la reproduction) est assujettie à sa politique d'utilisation que vous pouvez consulter en ligne.

https://apropos.erudit.org/fr/usagers/politique-dutilisation/ 


\title{
Travail, handicap et discrimination : lorsque le travail devient aussi un espace de production du handicap
}

\author{
Rachid Merzouk \\ Professeur, École de service social, Université d'Ottawa
}

Mais la discrimination négative ne consiste pas à donner plus à ceux qui ont moins, elle fait au contraire d'une différence un déficit marquant son porteur d'une tare quasi-indélébile. Être discriminé négativement, c'est être assigné à un destin sur la base d'une caractéristique que l'on n'a pas choisie, mais que les autres vous renvoient sous la forme d'un stigmate. La discrimination négative est une instrumentalisation de l'altérité constituée en facteur d'exclusion.

(Castel, 2007, p.12)

Au cours des trente dernières années, de nombreux progrès ont été réalisés en matière de reconnaissance des droits des gens que l'on désigne généralement comme "personnes handicapées » ou "vivant avec une incapacité ». Dans cet article, je parlerai plutôt de "personnes en situations de handicap ". Cette désignation présente plusieurs avantages. Elle permet de mettre l'accent sur le caractère situationnel du handicap en dépassant notamment l'amalgame qui existe encore entre maladie, déficience, incapacité et handicap. Ce caractère situationnel permet de comprendre que les personnes ayant une incapacité ne sont pas continûment handicapées (Goffman, 1975). Elles sont régulièrement confrontées à des environnements qui les mettent en situation de handicap. En lien avec cette idée, certains auteurs parlent de processus de 
"...les situations de handicap peuvent toucher différents secteurs de la vie d'une personne ayant une incapacité, d'où l'intérêt de parler de situations au pluriel. » production du handicap (Fougeyrollas, 1996). Enfin, mettre en lumière le caractère situationnel du handicap permet de montrer que les situations de handicap peuvent toucher différents secteurs de la vie d'une personne ayant une incapacité, d'où l'intérêt de parler de situations au pluriel.

L'un de ces secteurs est celui du travail. Dans ce domaine, quelqu'un peut être en situation de handicap lorsqu'il se trouve dans un environnement professionnel non adapté aux compétences particulières qu'il possède en tant que personne ayant une incapacité. Cet environnement inadapté peut à première vue résulter des barrières architecturales, mais les pratiques discriminatoires de la part des employeurs ou des autres employés peuvent également contribuer à mettre la personne ayant une incapacité en situation de handicap professionnel.

\section{Travail et handicap, quel enjeu pour la question du travail en général?}

"...des progrès ont été réalisés en faveur de l'inclusion dans la société canadienne des personnes en situations de handicap..."
L'intérêt accordé à la participation au marché du travail des personnes en situations de handicap tient en deux raisons principales. La première découle de la législation sur l'équité en matière d'emploi et sur les droits de la personne, qui assure l'accès au marché du travail aux personnes avec une incapacité. La deuxième, tout aussi importante, concerne le moyen d'accroître sur le marché du travail le nombre de personnes avec une incapacité. Dans ce cas, la motivation tient aux pénuries de main-d'œuvre et de travailleurs qualifiés qui résultent, entre autres, du vieillissement de la population. Dans cette perspective, maximiser l'activité des personnes avec une incapacité peut constituer une des manières de contrer cette pénurie imminente (Williams, 2006).

Dans cette perspective, des progrès ont été réalisés en faveur de l'inclusion dans la société canadienne des personnes en situations de handicap grâce à la législation fédérale qui a mis en place des mesures visant à faciliter l'accès, et puis le maintien, au sein 
"...la nécessité

même de légiférer

en la matière révèle

l'existence d'un réel

malaise dans le rapport

travail et handicap. " du marché du travail des personnes ayant une incapacité. Cette législation en faveur de l'emploi des personnes en situations de handicap s'est produite dans un contexte global du travail qui se caractérise par les trois facteurs suivants : il s'agit d'un passage, en l'espace des vingt dernières années, d'un contexte global d'une pénurie d'emploi à une pénurie annoncée de main-d'œuvre, d'une mondialisation du marché du travail et enfin d'une transformation radicale des conditions et des expériences du travail. C'est à la lumière de ces facteurs que l'on doit lire en partie les progrès législatifs en faveur de l'emploi des personnes en situation de handicap. En effet, les acteurs du marché de l'emploi et le législateur sont désormais conscients que la loi sur les droits de la personne leur impose d'assurer une égalité d'accès au travail pour les demandeurs d'emploi ayant une incapacité.

Cependant, la nécessité même de légiférer en la matière révèle l'existence d'un réel malaise dans le rapport travail et handicap. Il s'agit d'un rapport qui contribue au handicap et qui engendre une forme de souffrance sociale. Laquelle souffrance fait écho à d'autres souffrances pouvant être vécues par ailleurs par les personnes en situations de handicap. Le rapport de souffrance entre travail et handicap peut être nourri par la forme de violence que constitue l'attitude de discrimination de la part de l'employeur ou des collègues, ou encore par des échecs professionnels à répétition. Un rapport conflictuel de cette nature peut constituer finalement pour une personne le noyau dur des conséquences sociales de son incapacité physique.

Ces expériences récurrentes et marquées par l'échec favorisent l'exclusion, puisque les marges de manœuvre pour s'en préserver apparaissent de plus en plus réduites. Ces expériences sont alors le reflet d'un processus de discrimination qui caractérise le monde du travail d'aujourd'hui tel que le décrit Henri-Jacques Stiker :

L'entreprise, aujourd'hui plus que jamais, cherche ce que l'on appelle l'excellence. Excellence professionnelle certes, mais aussi dans la personnalité, les capacités relationnelles, le look extérieur. La passion de l'excellence rend avengle aux possibilités réelles des gens et à la 
bonne recherche de l'adéquation entre les qualités des personnes et les emplois concrets, non pas ceux que l'on met sur le papier, mais ceux réellement exercés. $\mathrm{La}$ discrimination consiste ici à écarter d'emplois possibles des personnes que l'on se représente comme inaptes, car cette passion de l'excellence et de la performance envahit tout le champ de conscience des recruteurs (2006, p. 41).

"...le monde du travail est aujourd'hui habité par un souci de l'excellence, dont le revers est l'amalgame qui, dans l'esprit de bon nombre de recruteurs, associe personnes avec incapacité à nonproductivité."

\section{"Le travail, et plus particulièrement la discrimination pour l'accès au travail, produit dans ces conditions le handicap..."}

On le voit bien, le monde du travail est aujourd'hui habité par un souci de l'excellence, dont le revers est l'amalgame qui, dans l'esprit de bon nombre de recruteurs, associe personnes avec incapacité à non-productivité. Cette confusion produit une situation sociale inéquitable, car discriminatoire, et conduit à minimiser le potentiel productif de la personne avec une incapacité, la plaçant alors effectivement en situation de handicap. Cette situation sociale, dans une causalité à double sens, se fonde sur des représentations sociales dévalorisantes de la capacité de travailler de la personne et alimente à l'égard de l'ensemble des personnes exclues du travail, parmi lesquelles celles avec incapacité, une vision qui les considère comme « inutiles parce que frappé[e]s du stigmate de l'inaptitude ou de l'inefficience" (Lhuillier, 2002, p.17).

Le travail, et plus particulièrement la discrimination pour l'accès au travail, produit dans ces conditions le handicap et contribue à l'installation de la personne avec une incapacité dans une situation de handicap qui résulte alors du fait que cette personne se trouve ainsi privée des fonctions que le travail assure ordinairement à son titulaire dans notre société.

En lien avec la question de la formation professionnelle des personnes ayant une incapacité, et se référant à la dernière enquête sur la participation et les limitations d'activités publiée par Statistique Canada (EPLA, 2001), l'analyste Cara Williams commente que :

Environ le tiers [33\%] des personnes [ayant une incapacité] occupées avaient au moins un certificat ou un diplôme postsecondaire, contre $23 \%$ des personnes 
"En tant que

lieu d'inégalité, le travail devient un espace propice à la discrimination envers les personnes ayant une incapacité puisqu'elles sont placées en compétition avec des personnes sans incapacité. " en chômage et $17 \%$ de celles ne faisant pas partie de la population active. Toutefois, ces proportions sont nettement inférieures à celles de la population générale âgée de 15 à 64 ans sans incapacité, dont $48 \%$ environ avaient au moins un certificat ou un diplôme postsecondaire (Williams, 2006, p. 21).

Si la question de la formation est cruciale par rapport au travail pour l'ensemble de la population active, il apparait clairement que le niveau moindre de formation des personnes ayant une incapacité constitue pour elles un obstacle supplémentaire dans leur parcours professionnel. Ce faible niveau de formation doit être analysé à la lumière des barrières qu'elles rencontrent à l'école et dans les établissements de formation en général.

Cependant, ce manque de formation n'empêche pas la majorité de ces personnes d'exprimer leur volonté de trouver un emploi ou de conserver celui qu'elles ont déjà. Alors que confrontées la plupart du temps au surcoût engendré par les moyens nécessaires à la compensation de leur incapacité, elles ne tirent pas nécessairement un revenu suffisant de l'emploi qu'elles occupent. L'intérêt premier du travail, dans le cas de ces personnes, n'est pas tant l'apport financier que l'obtention d'une reconnaissance sociale.

En tant que lieu d'inégalité, le travail devient un espace propice à la discrimination envers les personnes ayant une incapacité puisqu'elles sont placées en compétition avec des personnes sans incapacité. Cette inégalité se donne à lire à travers d'autres résultats publiés par Statistiques Canada à partir de l'EPLA, précédemment indiquée et selon laquelle "[1]e taux d'activité diminue à mesure que le degré d'incapacité augmente. Par exemple, le taux d'activité des personnes qui ont une incapacité légère était de $63 \%$, mais il est tombé à $28 \%$ chez celles qui ont une incapacité grave ou très grave " (Statistiques Canada, 2006, p. 3).

Cette inégalité, on le voit à travers ces chiffres, se traduit pour un grand nombre de ces personnes en un éloignement définitif du marché du travail, et donc de la vie active puisque la même enquête révèle que parmi les deux millions de personnes canadiennes âgées 
"L'histoire de Catherine illustre un processus de discrimination à multiples facettes. » de 15 à 64 ans et qui déclarent avoir une incapacité, à peine 45 $\%$ d'entre elles font partie de la population active, tandis que ce chiffre atteint les $80 \%$ pour les personnes sans incapacité (cité par Williams, 2006, p. 18).

\section{Le récit de vie comme méthodologie d'enquête}

Dans le domaine du travail, la personne ayant une incapacité vit la plupart du temps une double expérience d'exclusion : d'abord au moment d'accéder à un emploi, ensuite une fois qu'elle est en poste. L'objectif de cet article est de montrer que cette exclusion en deux temps et les processus qui y entrent en jeu doivent être analysés à la lumière des expériences d'exclusion et de discrimination que la personne aurait pu vivre, ou vit encore, dans d'autres secteurs de la vie. Pour illustrer cette idée, je vais me référer à l'histoire de Catherine (nom fictif) dont le récit est principalement articulé autour de ce qu'elle-même appelle «mes malheureuses expériences professionnelles ». Ces expériences sont malheureuses parce qu'elles font naitre chez elle des sentiments de rejet et d'échec qui ne sont pas sans lui rappeler ceux éprouvés dans d'autres circonstances.

L'histoire de Catherine présente l'avantage de concentrer à elle seule diverses expériences personnelles et professionnelles rapportées par plusieurs autres personnes dont j'ai recueilli les récits de vie pour ma recherche doctorale (Merzouk, 2008), dans le cadre d'une enquête empirique selon la méthode biographique de récit de vie (Gaulejac de et Legrand, 2008). L'histoire de Catherine fait apparaitre son "handicap " comme une série de situations de discrimination qu'elle fut amenée à affronter à différents moments de sa vie. Intéressons-nous donc de près à son parcours biographique et aux multiples expériences qu'elle décrit dans son récit.

\section{Le handicap, une expérience de multiples discriminations}

L'histoire de Catherine illustre un processus de discrimination à multiples facettes. Il s'agit d'une discrimination qui, se fondant 
"Le parcours familial de Catherine se caractérise par un refus de la part de ses parents de reconnaitre à la fois la réalité de son incapacité physique et de lui procurer par conséquent des moyens d'adaptation lui permettant de fonctionner au quotidien." sur la présence d'importantes limitations physiques de nature motrice, place Catherine en différentes situations d'exclusion. Le récit biographique fourni sur l'histoire de son handicap témoigne de plusieurs formes et espaces d'exclusion. Une exclusion vécue d'abord au sein de son propre milieu familial, puis une autre vécue dans le milieu scolaire et une troisième éprouvée dans le monde du travail.Voici un bref rappel de quelques éléments biographiques qui permettent de présenter le contexte familial et le contexte social qui caractérisent la situation de Catherine.

Catherine est née en 1968. Une naissance prématurée et inattendue durant les vacances. Lors de cette naissance entamée à domicile et poursuivie pendant le transfert à l'hôpital, elle manque d'oxygène. De cette naissance mouvementée, elle gardera de graves séquelles motrices, mais qui ne se révéleront qu'au cours de sa deuxième année. Sur la base d'un premier diagnostic, Catherine passera durant son enfance de longues périodes à l'hôpital et en maison de convalescence. C'est dans ce cadre institutionnel qu'elle acquiert les apprentissages de base comme la parole ou encore les rudiments d'une hygiène corporelle.

Dès l'âge de sept ans, elle sera contrainte de porter des chaussures orthopédiques. Mais sa démarche reste incertaine et les chutes sont très fréquentes. À l'âge adulte, elle se déplace en fauteuil roulant manuel et peut effectuer debout, non sans difficulté, quelques pas de marche en s'aidant d'une canne.

\section{La canne, un symbole de discrimination}

Le parcours familial de Catherine se caractérise par un refus de la part de ses parents de reconnaître à la fois la réalité de son incapacité physique et de lui procurer par conséquent des moyens d'adaptation lui permettant de fonctionner au quotidien. En rapport avec les années de son enfance, elle se souvient avoir participé à des activités sociales extérieures en compagnie de son oncle bien plus qu'avec ses propres parents. Elle affirme que cet oncle était plus proche d'elle et plus à l'écoute de ses besoins que ne l'étaient ses propres parents. C'est également avec la famille de cet oncle qu'elle partait le plus souvent en vacances pendant l'été. 
C'est pendant les vacances qu'elle s'aide pour la première fois d'une canne que son oncle lui fabrique à l'aide d'une branche d'arbre. Cette canne lui permet de réduire le risque de chute auquel elle était constamment exposée du fait de l'affaiblissement du contrôle volontaire du mouvement de certains muscles. Cet outil lui permet également d'agrandir son rayon de déplacement et de promenade. Cette "canne de vacances» est utilisée durant les jours de la semaine, puis est précautionneusement "rangée " la veille de l'arrivée de ses parents sur les lieux. Au lendemain de leur départ, elle est à nouveau sortie, par l'oncle.Voici le récit que Catherine fait de l'histoire de la canne:

"Je partais pratiquement tous les étés avec mon oncle et ma tante; mes parents venaient les fins de semaines. Alors, dans la semaine, mon oncle m'avait fait une canne avec une branche d'arbre. Toute la semaine, je me promenais partout sans tomber, sans rien. Le vendredi, avant que mes parents arrivent, on savait à peu près à quelle heure ils arrivaient, on cachait la canne pour la fin de semaine."

Ce jeu de dissimulation dure pendant trois étés de suite. C'est à l'occasion d'une arrivée-surprise de leur part que les parents découvrent l'existence de la canne et son utilisation par leur fille. Cette découverte est suivie d'une houleuse discussion entre d'un côté les parents et, de l'autre, les grands-parents et l'oncle. À aucun moment, Catherine n'est consultée, alors que c'est elle qui profite de la canne et en retire un sentiment de sécurité. Les chutes qui se produisaient de façon répétée étaient susceptibles d'engendrer chez elle une peur inhibitrice à l'égard de la marche. Finalement, c'est une médiation menée par la grand-mère qui permet à Catherine d'utiliser à la vue de tous une canne. Mais cette acceptation parentale en limitera l'utilisation aux déplacements dans la rue et ne s'appliquera pas à l'intérieur de la

"L'acceptation par les parents de l'utilisation d'une canne est incertaine..." maison. L'acceptation par les parents de l'utilisation d'une canne est incertaine : «C'était une acceptation pas complète, à tel point que quand la première canne a cassé,je me demandais s'ils allaient m'en acheter une autre [rires, suivis d'un silence] ». Cet épisode 
constitue pour Catherine une nouvelle source d'angoisse, puisqu'il est question de la préservation ou de la perte de l'autonomie acquise grâce à la canne.

Maintenant à l'âge adulte, et même si son incapacité s'est aggravée, Catherine déclare éviter d'utiliser sa canne lors de ses visites à ses parents. On le voit, le refus parental d'autoriser Catherine à utiliser une canne, et qui est repris ici à titre d'exemple parmi d'autres dans son récit biographique, révèle l'existence dans son histoire familiale d'une attitude de rejet de son incapacité physique. Cette attitude parentale ne semble pas tenir compte des besoins spécifiques de Catherine, notamment en matière d'adaptation.

Ces différents souvenirs et événements, non dénués d'une charge émotionnelle lors de leur évocation par Catherine, illustrent l'attitude de ses parents vis-à-vis de son incapacité physique. En effet, il s'agit de leur part d'une attitude de refus $\mathrm{du}$ handicap. Ce geste ne se fonde sur aucune recommandation médicale; il va à l'encontre non seulement de la volonté de la première intéressée, mais également à l'encontre d'un réel besoin de sécurité et d'autonomie dans ses déplacements.

Dans le parcours biographique de Catherine, le récit de ce qu'elle appelle «l'arrivée de la canne dans [sa] vie » est une belle illustration des comportements discriminatoires auxquels, très tôt, elle a dû faire face. En effet, la réalité familiale et la situation de contradiction qui en résulte ne constituent qu'une facette du processus répétitif de discrimination et d'exclusion qui caractérise son parcours biographique en général. En effet, il est un autre espace où Catherine fera l'expérience de la discrimination en lien avec son incapacité physique. Il s'agit de l'école et des relations avec les autres enfants de son âge tout au long de ses années au primaire.

\section{L'école, lieu des premières expériences de discrimination sociale}

À la suite d'une décision de ses parents qui refusaient de l'envoyer dans une école spécialisée pour enfants avec des besoins spéciaux, 
"...toutes ses années d'école seront marquées par une mise à l'écart du reste des élèves de sa classe et par un nombre important d'embûches de nature administrative et institutionnelle. »
Catherine est admise à l'école régulière de son quartier. Cette admission ne sera pas pour elle le signe d'une réelle insertion scolaire et sociale; en effet, toutes ses années d'école seront marquées par une mise à l'écart du reste des élèves de sa classe et par un nombre important d'embûches de nature administrative et institutionnelle.

À ses années d'école, est associée une situation d'isolement constant qui s'explique, à ses yeux, par le fait que "physiquement " elle n'était pas apte à "avancer au même rythme que les autres enfants".

À titre d'exemple, pendant la récréation, et sur instruction de son enseignante, elle reste dans la salle de classe en compagnie d'élèves désignés pour lui tenir compagnie. Cette mise à l'écart, sous prétexte de la protéger contre les bousculades dans la cour et de réduire ses efforts de déplacement, contribue à sa solitude et à son isolement par rapport aux autres enfants.

L'effet paradoxal de cette attitude surprotectrice est de rajouter à ses difficultés en la maintenant dans une situation d'exclusion de l'espace de socialisation constitué de la cour de récréation et des autres activités scolaires ou parascolaires. Cette même logique surprotectrice pousse les enseignants de Catherine, dont l'incapacité physique rend le mouvement des mains lent et imprécis, à faire accomplir à sa place tous les travaux pratiques par un autre élève.

Le souvenir de ses expériences d'exclusion à l'école conduit Catherine à exprimer dans son récit une ambivalence à propos de la pertinence de sa scolarisation à l'école régulière de son quartier, par opposition à une école spécialisée où elle aurait passé ses années primaires parmi d'autres enfants ayant eux aussi une incapacité physique. Elle considère ainsi que la fréquentation d'une école spécialisée lui aurait permis une meilleure insertion dans le groupe et l'aurait libérée de la solitude et de l'isolement qui ont marqué ses années d'école. Par contre, elle pense que cela se serait produit au détriment d'une certaine stimulation intellectuelle, dans la mesure où la scolarité dans les écoles spécialisées à son époque était principalement axée sur un apprentissage manuel, alors même qu'elle avait «mentalement toutes les possibilités ». 
On peut expliquer cette ambivalence par la contradiction radicale devant laquelle se trouve Catherine, entre ce qu'elle voudrait être et faire et la réalité de ce qu'elle vit. Il s'agit d'une situation de conflit accentuée par l'isolement social dans lequel elle a grandi. L'ambivalence qu' elle exprime la renvoie à une question de positionnement identitaire entre deux positions distinctes. Soit en tant qu'enfant comme les autres, sa place était à l'école régulière de son quartier, mais alors son isolement et sa mise à l'écart des autres enfants viennent lui rappeler qu'elle n'était pas à «sa " place. Soit en tant qu'enfant "différent ", elle aurait dû aller dans une école spécialisée parmi d'autres enfants ayant une incapacité. Dans ce deuxième cas, elle n'aurait pas pu poursuivre une scolarité poussée en vue de se diriger vers la profession qu'elle visait déjà : celle d'enseignante!

Finalement, Catherine termine, en milieu scolaire régulier, une scolarité qui la conduira jusqu'aux portes du marché de l'emploi, où l'attendent de nouvelles expériences de discrimination. C'est ainsi que la discrimination vécue dans son milieu familial, puis dans le milieu scolaire, se reproduira plusieurs années plus tard lorsque, en dépit de l'obtention d'un diplôme universitaire, elle devra rencontrer autour d'elle de nombreuses résistances vis-à-vis de son projet d'exercer la profession d'enseignante.

\section{Lorsque le parcours professionnel devient un parcours de discrimination}

Dès le départ, son intérêt pour l'éducation et l'apprentissage pousse Catherine à vouloir devenir enseignante. Dans cette perspective, elle s'inscrit à un programme universitaire de baccalauréat d'enseignement où elle réussit l'ensemble de ses cours. Cependant, une fois arrivée à la phase des stages pratiques, les obstacles se dressent sur son parcours. Ainsi rencontre-t-elle, dès son premier stage, une attitude réticente de la part des autres professionnels quant à sa capacité de prendre comme enseignante la responsabilité d'une classe d'enfants. Cette résistance rencontrée comme stagiaire dans le milieu scolaire ne provient pas des élèves, mais des enseignants. Dans son récit, elle décrit cette première 
"...malgré sa réussite dans les études menant à la profession d'enseignante, la principale barrière qui s'opposera à la réalisation de son insertion professionnelle sera l'attitude discriminatoire des formateurs. " expérience de discrimination par rapport au travail de la façon suivante :
"C'était quand même pas facile;j'ai réussi,j'aurais pu enseigner, je n'avais pas de difficultés avec les enfants; encore là, la difficulté venait des adultes: "Tu peux être dangereuse!”, "Tu ne peux pas!”. Donc, j'ai un beau diplôme universitaire qui est au fond d'un tiroir et qui ne sert à rien [silence]. "

On le voit bien, malgré sa réussite dans les études menant à la profession d'enseignante, la principale barrière qui s'opposera à la réalisation de son insertion professionnelle sera l'attitude discriminatoire des formateurs. Malgré les barrières de nature matérielle et architecturale auxquelles s'ajoutait la réticence de ses formateurs et de ses collègues, Catherine fait preuve de ténacité et ne ménage aucun effort pour ne pas renoncer à la poursuite de sa formation universitaire.

Après l'obtention de son baccalauréat et à la fin de ses premières expériences professionnelles de terrain à travers les stages, ses efforts se poursuivent lors des démarches comme candidate à un poste d'enseignante. À cette étape de son parcours professionnel, elle est confrontée à une attitude discriminatoire de la part des employeurs qui s'abritent derrière le leitmotiv de la sécurité des élèves. Elle vit en effet de nouvelles épreuves lors des entrevues d'embauche quand les questions des employeurs sont dirigées principalement vers les différents aspects de ses incapacités physiques, plutôt que sur ses compétences proprement professionnelles. Parlant de ces différentes entrevues, elle raconte avoir le sentiment que sa candidature était perçue comme celle "d'une personne essentiellement handicapée ", c'est-à-dire porteuse d'un manque. Tandis qu'elle estime pour sa part avoir suivi la formation adéquate et avoir développé les compétences professionnelles nécessaires pour enseigner.Voici un extrait relatant son parcours professionnel à cette époque :

"Il y a même des professeurs, au moment où j'étais en formation, je me souviens, ils m'ont dit : "Qu'est-ce 
que tu viens faire dans une école, c'est pas ta place ici" [...] C'est pas par ce que je n'avais pas les capacités pour enseigner. [...] Une entrevue d'embauche pour un enseignement, qui a duré environ trente minutes, on a passé vingt minutes à parler de mon handicap : "Qu'est-ce que tu vas faire si?", "Comment tu fais si?”, sans rapport avec la capacité d'enseigner. [silence] Ils voulaient pas que j'enseigne, c'est tout! ".

"Qu'est-ce que tu viens faire dans une école, c'est pas ta place ici! [...] Ils voulaient pas que j'enseigne, c'est tout! »

Pourtant, on peut aisément observer que l'obtention d'un diplôme universitaire est une réalisation qui est en soi la meilleure garantie de la détermination et de la motivation de la personne pour la profession à laquelle conduit le diplôme en question. En effet, cette détermination est à analyser à la lumière des très nombreux obstacles qu'il est nécessaire de franchir pour arriver jusqu'à l'université et réussir son baccalauréat, en particulier pour une personne avec une incapacité. C'est pourquoi il est plus qu'étonnant que de nombreux employeurs ne voient pas dans le profil de la personne candidate ayant une incapacité celui d'une éventuelle employée qui est apte à s'investir pleinement dans son emploi, décroché au bout de tant d'efforts et de persévérance.

Ce constat s'applique parfaitement au parcours professionnel de Catherine. En dépit de la ténacité dont elle a fait preuve dans la poursuite de ses études, puis dans ses nombreuses démarches pour décrocher un poste d'enseignante, ses qualités et sa détermination

"...candidature

d'une personne avec une incapacité est principalement perçue en termes de manque de productivité, d'assiduité et même de sécurité. » ne semblent pas retenir l'intérêt des recruteurs, alors que ce sont là les caractéristiques mêmes que tout employeur souhaite rencontrer chez les membres de son personnel.

En reprenant l'interprétation fournie par Catherine à partir de ses propres expériences, la candidature d'une personne avec une incapacité est principalement perçue en termes de manque de productivité, d'assiduité et même de sécurité. Autrement dit, la visibilité de l'incapacité occulte les qualités personnelles 
et professionnelles qui peuvent être présentes chez la personne candidate. Tout se passe comme si la présence d'une incapacité physique ou intellectuelle enclenche un mécanisme incontrôlable de mise en jeu d'un ensemble de représentations sociales (Jodelet, 1984) au sujet du handicap et des personnes avec une incapacité. Ces représentations sociales légitiment aux yeux des recruteurs leur attitude réticente qui devient alors discriminatoire à l'égard de la candidature de la personne ayant une incapacité.

Ce récit illustre comment les représentations sociales répandues dans l'imaginaire de notre société à propos du handicap alimentent des stéréotypes chez les employeurs et les autres employés envers les personnes ayant une incapacité. Ces stéréotypes finissent par constituer un frein considérable à l'insertion professionnelle de ces personnes, d'autant plus que ces mêmes stéréotypes s'appuient sur certaines limitations objectives que le manque d'accessibilité architecturale notamment - impose à la personne. Ce manque d'accessibilité, qui peut être dû également à l'organisation du milieu du travail, empêche la personne de fonctionner à sa pleine capacité. À cela s'ajoute l'effet auto-accomplissant de certaines prophéties (Wilkins, 1976), c'est-à-dire, des situations où la personne ayant une incapacité, à force d'y être confrontée et privée de toutes les chances de démontrer le contraire, finit par se conformer aux attentes négatives et aux stéréotypes exprimés envers elle. Dans le cas d'une personne régulièrement confrontée à ces stéréotypes, l'estime de soi est violemment atteinte et le sentiment d'inutilité est brutalement restauré. La peur est reprise à son compte par la personne ayant une incapacité et finit par produire chez elle un effet de blocage et d'autocensure.

Les stéréotypes en question ici sont en lien avec la perception de l'incapacité uniquement en termes de manque : le manque de productivité, l'absentéisme, le manque de sécurité. Mais ils peuvent pareillement véhiculer l'image d'une personne employée ayant une incapacité dont la présence nuirait à l'image de l'entreprise, à l'esprit d'équipe au travail ou encore pourrait engendrer une surcharge de travail pour les autres employés, ses collègues. Finalement, ces stéréotypes font apparaître aux yeux de l'employeur une sorte d'incompatibilité entre le profil type de la 
"La confrontation à ces stéréotypes, de façon récurrente exerce sur la personne ayant une incapacité un effet de violence..." personne à employer et celui de la personne avec incapacité qui se présente pour le poste.

La confrontation à ces stéréotypes, de façon récurrente exerce sur la personne ayant une incapacité un effet de violence qui est déstabilisant parce qu'il vient en contradiction avec les espoirs nourris par elle, et par son entourage proche, au sujet de son avenir professionnel. Ces espoirs peuvent avoir été alimentés, à titre d'exemple, par une précédente réussite dans les études, l'obtention d'une formation qualifiée, ou encore par les programmes mis en place par les universités afin d'augmenter la proportion de leur population étudiante ayant une incapacité. L'effet de violence en question surgit au moment des premières expériences sur le terrain, lors des stages, ou encore au moment de la recherche du premier emploi. À cette étape, l'effet de déception peut conduire la personne jusqu'à la remise en question de l'utilité de ses précédents efforts, et même de la légitimité de ses attentes face à l'emploi visé et au travail en général.

Dans le cas de Catherine, les expériences répétitives de discrimination qu'elle traverse lors de ses recherches d'un poste d'enseignante finissent par la conduire, ainsi que son entourage, à remettre en question la pertinence du choix qui fut le sien d'entreprendre des études universitaires. Elle décrit ainsi cette remise en question :

"Ils [es proches] doivent se dire qu'elle s'est battue jusqu'à l'université, c'est quand même pas à la portée de tout le monde. Même en n'ayant pas de handicap, c'est pas tout le monde qui se rend jusqu'à l'université. D'abord, ils ont vu que j'ai fourni des efforts, aussi bien physiquement, moralement, intellectuellement, mais ça mène nulle part; ils se disent : "est-ce que ça valait la peine qu'elle le fasse?" [...] Ils doivent voir que je me suis battue, mais que ç'a rien donné de plus! "

Cette remise en question de la validité et de l'utilité de son choix de faire des études universitaires, qui étaient sensées la mettre à l'abri de la précarité sur le marché du travail, est non seulement 
"Au bout de plusieurs candidatures à

des postes dans ce domaine, dont aucune ne fut couronnée de succès, et découragée par l'attitude réticente de la part des commissions scolaires et des autres enseignants à la fois, Catherine renonce à l'enseignement et s'oriente vers d'autres possibilités d'emploi." l'expression d'une déception profonde, mais aussi d'un début de lassitude et de recherche de nouvelles possibilités professionnelles, sans lien avec l'enseignement.

Au bout de plusieurs candidatures à des postes dans ce domaine, dont aucune ne fut couronnée de succès, et découragée par l'attitude réticente de la part des commissions scolaires et des autres enseignants à la fois, Catherine renonce à l'enseignement et s'oriente vers d'autres possibilités d'emploi. Sa décision s'explique également par des contraintes économiques et par son désir d'atteindre une certaine autonomie financière.

De ce fait, Catherine se tourne vers des programmes sociaux destinés à encourager l'employabilité des personnes en situations de handicap. Au Canada, ces programmes offrent à l'employeur de nombreux avantages fiscaux, telles une prime à l'emploi ou encore la déduction pour frais de modification de l'organisation et de l'espace de travail afin de réduire les barrières pouvant restreindre l'activité professionnelle de la personne employée et qui présente une incapacité.

Relevant d'une politique générale en faveur des personnes en situations de handicap, ces programmes se révèlent utiles pour le maintien en activité d'une personne dont l'incapacité est apparue en cours de carrière, ou encore pour faciliter l'acquisition d'une première expérience professionnelle qui permet à la personne de faire "ses preuves » aux yeux d'un futur employeur. Limitée cependant aux premiers mois de l'embauche, leur durée d'application, peut produire un effet pervers de leur utilisation par certains employeurs peu scrupuleux.

En tant que bénéficiaire de ces programmes, Catherine occupe successivement plusieurs emplois dans différents secteurs professionnels.Aucun de ces emplois n'a de lien avec sa formation universitaire ou le domaine de l'enseignement. Malgré le fait qu'il s'agisse de tâches de secrétariat ou de bureautique qui exigent une formation scolaire moins élevée que la sienne, Catherine n'a jamais pu conserver un emploi pendant plus de six mois. En dépit de la modestie de ses attentes vis-à-vis des conditions de maintien en poste, ses espoirs sont systématiquement déçus. 
"Cette instabilité

professionnelle vécue par Catherine s'explique par l'absence de scrupules de certains employeurs qui gardent en emploi la personne ayant une incapacité le temps que durent les avantages fiscaux obtenus lors de son embauche."
Pourtant, elle se montre constamment disposée à occuper un emploi, même à temps partiel. Par ailleurs, de tous les emplois qu'elle a occupés, elle garde le souvenir de la satisfaction exprimée par son employeur à l'égard de son travail et de l'accomplissement des tâches inhérentes au poste. Pendant six mois, elle se décrit comme "une employée idéale » avec "une auréole au-dessus de la tête ». Mais une auréole qui se perd mystérieusement, aux yeux de son employeur, passé le sixième mois. Cette période correspond aussi à la durée légale des avantages fiscaux que les programmes sociaux offrent à l'employeur.

Cette instabilité professionnelle vécue par Catherine s'explique par l'absence de scrupules de certains employeurs qui gardent en emploi la personne ayant une incapacité le temps que durent les avantages fiscaux obtenus lors de son embauche. Puis, ils la licencient pour embaucher une autre personne ayant une incapacité, et donc bénéficier d'une nouvelle période d'avantages fiscaux.

Ce procédé révèle à la fois les failles et les effets pervers de certains programmes qui touchent à l'emploi des personnes ayant une incapacité. Gail Fawcett présente un autre exemple de faille à travers le commentaire suivant qui est repris d'une personne ayant une incapacité : "Pour obtenir un emploi, il faut prouver à un employeur qu'on peut travailler. Cependant, pour survivre lorsqu'on n'a pas d'emploi, il faut convaincre le gouvernement qu'on ne peut pas travailler! " (Fawcett, 1999, p. 9).

Comme on peut l'observer, aux côtés de l'utilisation peu scrupuleuse par certains employeurs des programmes en faveur de l'emploi des personnes ayant une incapacité, d'autres failles dans ces mêmes programmes contribuent à l'effet de violence que peuvent vivre ces personnes sur le marché du travail et dans leur quête d'un emploi stable. Ainsi, tout au long de son parcours professionnel, chaque nouvelle embauche fait-elle naitre en Catherine de nouveaux espoirs d'un emploi permanent, rendant de ce fait la décision de rupture de contrat encore plus déstabilisante. Mais sa ténacité et sa force de volonté de vivre une réelle intégration professionnelle la conduisent à nourrir de nouveaux espoirs lors de chaque nouvelle embauche. Il 
"Face à l'attitude de l'employeur et aux violences qu'elle occasionne, il est très difficile de préserver une image positive de soi.» s'agit d'espoirs que cette embauche puisse déboucher sur son maintien en emploi à l'issue de la période initiale prévue par la loi. Ces espoirs sont par ailleurs alimentés par les possibilités que certains employeurs ne manquent d'évoquer pour elle durant les six premiers mois. Catherine se consacre alors corps et âme à son travail. Elle rapporte qu'elle se donne à «110\% de [son] potentiel ». Elle explique également qu'après coup, ces conditions font que l'emploi devient émotionnellement très exigeant. C'est «vidant émotionnellement! ", raconte-t-elle.

Cette exigence émotionnelle constitue une épreuve supplémentaire et un obstacle de plus dans la trajectoire de Catherine, non seulement parce qu'effectivement elle s'investit de toutes ses forces physiques et psychiques dans son travail et dans la réalisation des tâches qu'on lui confie, mais aussi parce que chaque décision de non-renouvellement de son contrat vient porter un coup à son estime de soi et à la considération qu'elle a pour elle-même. Face à l'attitude de l'employeur et aux violences qu'elle occasionne, il est très difficile de préserver une image positive de soi. Bien au contraire, la perte de l'emploi est source de dévalorisation de l'image de soi.

Confrontée à toutes ces douloureuses expériences professionnelles et sociales, Catherine ne peut s'empêcher en fin de compte d'éprouver un sentiment d'échec dont elle finit par s'auto-attribuer la responsabilité. Enfin, si toutes ses tentatives pour trouver un emploi durable et fixe n'ont pas abouti, ce serait bel et bien «à cause d'elle et de sa faute à elle ». Ce sentiment de culpabilité fait naitre une attitude de renoncement puisque finalement, c'est elle qui est " responsable » de sa propre situation socioprofessionnelle. Un renoncement qui oscille entre espoir et désespoir.Voici la façon dont Catherine interprète cette réalité :

"Comme ça fonctionne pas, on est un peu tanné de se battre. Mais on continue quand même de chercher. [...] À chaque fois, on a de l'espoir. Mais l'espoir déçu, ça devient comme de l'échec, pas un échec dû̀ à nous, mais on le perçoit comme un échec. Moi, ça me fait l'effet d'un mur. J'essaie de le traverser, mais à un moment 
donné, quand il $y$ a pas de porte, quand il $y$ a rien, on se tanne de taper dans un mur, [rire] à moins d'être complètement idiot là. Et donc, à ce moment-là, on se rabat sur le bénévolat et autres activités, parce que là, quand on est bénévole, il $y$ a pas de problème, on est extraordinaire à ce temps-là. »

S'il est exact que ces expériences récurrentes font de la situation socioprofessionnelle de Catherine une situation précaire et instable, les fréquents changements de lieux et des équipes de travail ont des conséquences sur les échanges sociaux et affectifs auxquels elle peut aspirer. Ces fréquents changements la privent effectivement de la possibilité de développer des échanges sociaux et affectifs en rapport avec son âge et ses aspirations personnelles. En effet, on le sait, les contacts permanents entre collègues de travail forment généralement un vivier pour l'établissement et la consolidation de relations sociales, voire amicales, en dehors du travail. À tel point que les fréquentes ruptures dans ces contacts à la fin de la période des six mois créent dans la vie de Catherine un processus de ruptures répétitives. En perdant son emploi, elle perd par là même les rares échanges sociaux auxquels elle peut prendre part en compagnie de ses collègues de travail.

Face à ces fréquents points de rupture (Castel, 2003), psychiquement douloureux puisqu'ils viennent régulièrement lui rappeler sa situation d'exclue, Catherine tente d'adopter une stratégie défensive qui consiste à «ne pas s'attacher aux gens" avec qui elle travaille. Mais elle prend vite conscience de la difficulté de se garder volontairement de nouer toute attache avec les membres de son équipe de travail. Se priver du moindre attachement relationnel et affectif vis-à-vis de ceux et de celles de ses collègues avec qui elle pouvait trouver des centres d'intérêt communs, Catherine n'y parvient pas; ce qui rajoute à ses difficultés affectives de quitter chaque emploi à l'issue des six premiers mois. C'est pourquoi, en écho à la violence des licenciements et des séparations répétitives qu'elle subit, Catherine se fait violence elle-même, en tentant de s'interdire tout lien affectif, afin de se protéger des conséquences socio-affectives de 
"...l'exclusion $d u$ travail s'accompagne ici d'un isolement relationnel qui est encore plus lourd du fait de l'appauvrissement de son réseau social à cause de ses limitations physiques qui l'empêchent de participer à des activités sociales à l'extérieur de son travail." sa réalité professionnelle. De toute évidence, l'exclusion du travail s'accompagne ici d'un isolement relationnel qui est encore plus lourd du fait de l'appauvrissement de son réseau social à cause de ses limitations physiques qui l'empêchent de participer à des activités sociales à l'extérieur de son travail.

Finalement, de même qu'elle a dû renoncer à la profession d'enseignante, et devant les promesses de reconduite de contrats non tenues, et par conséquent devant les licenciements répétitifs, Catherine finit par cesser toute recherche d'emploi rémunéré et se retourne vers le bénévolat pour accomplir pourtant des tâches identiques à celles qu'on lui confiait lorsqu'elle occupait un emploi salarié. Dans un contexte de bénévolat, la longévité des contrats et des missions remplis par elle atteste de ses capacités et compétences à réaliser le travail qu'on lui confie et à faire preuve de compétence et de savoir-faire. Ce qui montre que ce n'est pas là que se trouve l'explication du non-renouvellement de ses précédents contrats en tant que salariée.

"Les personnes avec un handicap c'est quelque chose, ça dérange, parce que ça entre pas dans le moule fin, intelligent, productif sans limites " (Catherine).

\section{Le handicap dans le monde du travail}

Le témoignage de Catherine illustre bien la problématique plus générale décrite au début de cet article portant sur le handicap dans le contexte du travail. Dans Les métamorphoses de la question sociale (1995), Robert Castel distingue trois fonctions du travail. La première concerne un processus de valorisation : la personne est valorisée à ses propres yeux et aux yeux de la société lorsqu'elle travaille et du fait de ce qu'elle produit par son travail. La deuxième fonction concerne le fait que le travail constitue le fondement des biens sociaux entre l'employeur, la personne travailleuse et la personne consommatrice du produit du travail. Enfin, la troisième fonction porte sur le travail comme moyen de redistribution des richesses. 
"Dans le cas d'une personne ayant une incapacité, travailler c'est aussi une façon de se prouver, et de prouver aux autres, qu'elle est tout aussi capable qu'une personne sans incapacité. "
Le rappel de ces trois fonctions sociales du travail permet de comprendre la portée des propos de Catherine lorsqu'elle déclare que « la principale conséquence sociale de [son] handicap, c'est au niveau du travail qu' [elle] la vit ». Cette "principale conséquence " de son incapacité physique se traduit par une atteinte à son statut social. En effet, c'est par son travail, lorsque celui-ci n'est pas précarisé, que toute personne employée obtient non seulement son autonomie financière, mais aussi une reconnaissance sociale. Dans le cas d'une personne ayant une incapacité, travailler c'est aussi une façon de se prouver, et de prouver aux autres, qu'elle est tout aussi capable qu'une personne sans incapacité. Ici, le travail est recherché, même lorsqu'il ne procure pas un revenu élevé ni même suffisant, car il assure quand même l'accès à une identité personnelle et à une identité sociale qui sont valorisées.

Quant au niveau de la scolarité, dans un marché du travail qui est sélectif et exigeant en diplôme, les personnes en situations de handicap, du fait souvent de leur manque de formation, sont réduites à des emplois à faibles revenus. Comme on l'a vu à travers le parcours de Catherine, les différents postes de travail qu'elle a pu occuper la mettaient en situation de surqualification puisque sa formation universitaire la qualifiait à occuper un poste d'enseignante dont les exigences professionnelles sont plus élevées que celles des emplois qu'elle a obtenus. Mais il n'en va pas de même pour la plupart des personnes ayant une incapacité et qui sont en emploi ou à la recherche d'un travail.

L'injonction d'excellence qui domine le monde du travail n'est pas uniquement perceptible pour les chercheurs préoccupés par ses dernières évolutions (Stiker, 2006; et autres), car les personnes confrontées elles-mêmes de très près à cette exigence en prennent régulièrement conscience. C'est ainsi que Catherine explique spontanément ses "malheureuses expériences professionnelles » par le «le règne de la super productivité » :

"Il faut être efficace, rapide, beau, fin, intelligent, meilleur que tout le monde. Je pense que c'est ça. Pas en tout, peut-être... Pas la seule partie du problème, mais je crois que c'est une grosse part, la 
"Dans cette perspective, et parce qu'il est mû par la logique de l'excellence et de la productivité, le travail contribue à donner sens au handicap et aux situations de handicap. " super production. [...] Quand il y a un emploi, [...] le patron, [...] il prend le meilleur. Il va pas essayer de prendre la personne handicapée qu'il sait que bon, bah elle a besoin d'aide... elle est moins rapide, elle a des contraintes".

Dans cette perspective, et parce qu'il est mû par la logique de l'excellence et de la productivité, le travail contribue à donner sens au handicap et aux situations de handicap. Le handicap résulte ici de la présence de la personne ayant une incapacité dans un milieu de travail qui est inadapté à ses compétences particulières et qui ne tient pas compte de ses besoins spécifiques.

$\mathrm{Au}$ sens de la loi sur le travail, et parce qu'elle était disposée à occuper un emploi Catherine appartenait bel et bien au moment de l'enquête à la population active canadienne (Statistique Canada, 2006), mais dans les faits elle était exclue du travail, puisqu'en dépit de sa formation elle peinait à obtenir un emploi qui soit stable et permanent. De plus, son parcours professionnel permet de montrer que le diplôme et le niveau de formation avancée ne mettent pas la personne ayant une incapacité à l'abri des discriminations qui se produisent à son encontre dans le marché du travail. Et alors que l'on s'accorde à reconnaitre que les exigences du marché du travail en termes de diplômes sont plus élevées aujourd'hui qu'elles ne l'étaient il y a 20 ou 30 ans, dans le cas des personnes en situations de handicap la visibilité de leur incapacité semble enclencher les mécanismes de discrimination à leur égard. Soit que ces mécanismes les excluent strictement du travail, soit qu'ils leur imposent des conditions de travail qui ne sont à la hauteur ni de leurs compétences, ni de leurs diplômes, et encore moins de leurs plans de carrière.

Les expériences de discrimination vécues à répétition par Catherine constituent à ses yeux, rappelons-le, le noyau dur des conséquences sociales de son "handicap ". Cela apparaît formellement, à travers une grande partie de son témoignage consacrée à la description de ses échecs professionnels ainsi que dans l'émotion qui sous-tend cette dernière. 
"Pour Catherine, ces multiples expériences prennent le sens d'un "gâchis ».»
Pour Catherine, ces multiples expériences prennent le sens d'un "gâchis ". Un gâchis notamment au regard de tous les efforts - physiques, moraux et intellectuels - qu'elle dit avoir fournis en vue de la poursuite, et de la réussite, de ses études universitaires. Mais aussi, un gâchis à la lumière de ce qu'elle possède comme compétences professionnelles et de sa disposition à se consacrer pleinement à son travail.

Dans une organisation professionnelle prônant la performance et la productivité comme les critères nécessaires pour occuper et garder un emploi, leur perturbation chez une personne ayant une incapacité est vécue par elle comme un échec personnel, lequel devient l'élément principal de son identité sociale. Et du fait de sa valeur négative, cette composante identitaire génère une souffrance chez la personne en situations de handicap.

Parce qu'il remet en question la confiance en soi, cet échec marque l'identité de la personne en situations de handicap au sceau de l'invalidation et de la stigmatisation. Il lui est alors difficile de conserver une image positive d'elle-même, alors même que la revalorisation et la reconnaissance sont des composantes identitaires déterminantes pour prétendre à une place autre que celle véhiculée par les stéréotypes au sujet des personnes ayant une incapacité. Ces stéréotypes conçoivent les personnes ayant une incapacité simplement en termes de manque.

\section{Discussion}

L'histoire de Catherine emprunte à celle de plusieurs autres personnes qui, comme elle, sont placées en situations de handicap. En ce sens, le parcours de cette femme constitue une forme de condensation du vécu d'autres personnes, au point que son histoire peut être vue comme un idéaltype de ce que peut être le parcours d'une personne ayant une incapacité face à l'emploi. En tant qu'idéaltype, son parcours peut représenter des variations comparativement à celui d'une autre personne, mais dans sa trame de fond, le vécu de Catherine illustre comment la présence d'une 
"La validité de

l'interprétation

formulée dans cet

article réside dans

la capacité d'autres

personnes dites

handicapées de s'y reconnaître et d'y reconnaître quelque chose de ce qu'elles vivent elles-mêmes, sans perdre de vue la singularité de chaque vécu. » incapacité chez la personne peut servir de facteur déclencheur de multiples expériences de discriminations.

Il ne s'agit pas de tenir ici, à partir des détails particuliers de l'histoire de Catherine, des propos qui auraient une portée générale et qui seraient ipso facto applicables à la réalité de toute personne ayant une incapacité et mise en situations de handicap. La validité de l'interprétation formulée dans cet article réside dans la capacité d'autres personnes dites handicapées de s'y reconnaitre et d'y reconnaitre quelque chose de ce qu'elles vivent elles-mêmes, sans perdre de vue la singularité de chaque vécu. Les personnes en situations de handicap constituent un groupe hétérogène, mais qui se caractérise, comme d'autres groupes sociaux, par une participation limitée à la société et au travail. Cette participation réduite peut sembler s'expliquer par les incapacités, physiques ou intellectuelles, que présentent les personnes dites handicapées. Toutefois, on néglige souvent de reconnaitre que ces incapacités ne deviennent un obstacle à la participation au travail que lorsque l'environnement est insuffisamment adapté aux besoins spécifiques de ces personnes. Cet environnement n'est pas fait que d'architecture. Il est fait aussi de préjugés, de stéréotypes et de représentations sociales qui véhiculent l'idée que les personnes avec une incapacité sont des personnes inaptes au travail, en particulier au travail rentable et productif.

Le handicap, par rapport au travail, résulte ainsi du refus de la société de prendre en compte dans son organisation la figure d'altérité que représente la personne avec une incapacité. Comme on l'a vu à travers l'histoire de Catherine, la personne ayant une incapacité peut être amenée à faire l'expérience de ce refus non seulement dans un monde de travail assoiffé de productivité et d'avantages fiscaux, mais aussi dans la famille ou à l'école.

C'est toute l'histoire de Catherine qui est affectee par les conséquences de son incapacité motrice et par de multiples expériences de discrimination qui la placent en situations de handicap. Toute son existence, dans ses différentes étapes, est touchée par l'exclusion. La place qui lui a toujours été assignée en est une de rejet. La double discrimination qu'elle vit par rapport 
à l'accès et ensuite au sein de son travail la renvoie aux autres stigmatisations qu'elle a subies dans le passé.

C'est pourquoi, en s'intéressant à la question des discriminations au travail à l'égard des personnes ayant une incapacité, il importe de ne pas perdre de vue que ces expériences sont à analyser à la lumière des précédentes discriminations vécues dans d'autres secteurs que le travail.Les expériences de discrimination et de rejet vécues en lien avec le travail agissent sur la personne en résonnance avec des expériences antérieures de semblable nature.

L'ensemble de ces expériences de discriminations apparait à ce stade comme une métasituation, c'est-à-dire une situation qui affecte l'ensemble du vécu et du parcours biographique de la personne ayant une incapacité. Il s'agit en effet d'une situation, ou plus justement de plusieurs situations qui se répètent au cours de l'histoire d'une personne, où sont réunis un ensemble de facteurs liés les uns aux autres. De nature psychosociale et psychoaffective, ces facteurs se conjuguent de manière à ce que la personne franchisse les barrières de son handicap et le dépasse, ou au contraire, de façon à ce que la personne reste prisonnière d'une réalité faite de contradictions et de discriminations. L'accès au travail, et les barrières qui peuvent s'y opposer pour une personne ayant une incapacité n'échappent pas à cette observation. L'inclusion ou l'exclusion du travail, et la reconnaissance sociale qui s'y rattache, s'inscrivent dans la trajectoire biographique de la personne. Elles le font en résonnance avec d'autres événements ou expériences précédentes d'exclusion et de mise à l'écart.

\section{Conséquences pour l'intervention sociale}

Une intervention sociale en faveur de la personne ayant une incapacité pour son accès, ou son maintien au travail ne peut pas faire l'économie de l'ensemble des expériences de discrimination qui jalonne le parcours biographique de cette personne. La question face à laquelle se trouve alors l'intervention sociale est la suivante : comment donner sens, en relation avec la problématique 
du travail, à l'articulation des composantes personnelles et familiales d'un côté et des composantes professionnelles de l'autre côté qui sont liées à l'expérience d'exclusion du travail dans le cas d'une personne ayant une incapacité?

Une telle recherche de sens ne saurait connaitre le succès si elle n'est pas conduite de pair avec la promotion, à l'échelle de l'ensemble des secteurs de la société, d'une politique d'égalité des chances et de non-exclusion. Cette politique passe non seulement par des campagnes d'information et de sensibilisation, mais aussi par le renforcement des mesures de discrimination positive, laquelle viendrait alors en contrepoids de la discrimination négative subie sur le marché du travail par les personnes ayant une incapacité. Parmi ces mesures, il faut continuer de compter les aides et primes à l'emploi qui sont l'expression légale d'une discrimination positive tout en en corrigeant certains effets pervers. C'est dans l'esprit de soutien à cette politique de discrimination positive que s'inscrit la position exprimée par l'historien et anthropologue Henri-Jacques Stiker lorsqu'il écrit :

Nous voulions pour terminer dire que la discrimination positive, au moins sous la forme de dispositions particulières pour les personnes handicapées et pour l'emploi, atteste une réelle efficacité. Ce qui veut dire tout simplement que la non-discrimination, principe fondateur et horizon indispensable, peut avoir besoin, pour se réaliser, des chemins de la compensation et des mesures de rétablissement d'une égalité citoyenne (2006, p. 41).

Finalement, il va sans dire que le rétablissement d'une telle égalité citoyenne reste conditionné au changement de regard de la société globale sur le handicap et sur les personnes ayant une incapacité et qui sont régulièrement mises, par l'effet de ce regard, en situations de handicap.

À ce changement de regard, le service social se doit d'apporter immanquablement sa propre contribution! 


\section{Bibliographie}

CASTEL, Robert (2007). La discrimination négative : citoyens ou indigènes?, Paris, Éd. Seuil.

CASTEL, Robert (2003). L'insécurité sociale. Qu'est-ce qu'être protégé?, Paris, Le Seuil/La République des idées.

CASTEL, Robert (1995). Les métamorphoses de la question sociale, Paris, Fayard.

DE GAULEJAC,Vincent, et Michel LEGRAND (sous la direction de) (2008). Intervenir par le récit de vie, Paris, Érès.

FAWCETT, Gail (1999). «Vivre avec un handicap sur le marché du travail : obstacles et solutions », Perceptions CCDS, Vol. 23, No 3, p.9.

FOUGEYROLLAS, Patrick, et Kathia ROY (1996). "Regard sur la notion de rôles sociaux. Réflexion conceptuelle sur les rôles en lien avec la problématique du processus de production du handicap ", Service social, 45 (3), p. 31-54.

GOFFMAN, Erving (1975). Stigmate : les usages sociaux des handicaps, Paris, Éditions de Minuit.

JODELET, Denise (1984). "Représentations sociales : phénomènes, concepts et théorie ", sous la direction de Serge Moscovici, Psychologie sociale, Paris, PUF, p. 357-378.

LHUILliER, Dominique (2002). Placardisés. Des exclus dans l'entreprise, Paris, Le Seuil.

MERZOUK, Rachid (2008) - Le sens de l'expérience sociale du handicap; une quête de sens. Pour une sociologie clinique du handicap, Doctorat de sociologie du Pouvoir, Université Denis Diderot Paris 7.

OFFICE DES PERSONNES HANDICAPÉES DU QUÉBEC (2006). « Les approches adaptatives et inclusives visant l'intégration scolaire, professionnelle et sociale des personnes handicapées ", Précisions de l'Office des personnes handicapées du Québec sur le concept d'intégration sociale et les approches inclusives, Drummondville, Québec.

REVUE ÉDUCATION PERMANENTE (2003). "Les “inemployables” ", No 156, 217 p.

STATISTIQUE CANADA (2006). L'emploi et le revenu en perspective, Vol. 7, N² 2, Ottawa.

STIKER, Henri-Jacques (2006). «Les personnes en situation de handicap dans l'entreprise ", Reliance, No 19 , p. 34-41.

WILLIAMS, Cara (2006). "L'incapacité en milieu de travail », dans Statistique Canada, L'emploi et le revenu en perspective, Ottawa.

WILKINS,William E. (1976). «The Concept of a Self-Fulfilling Prophecy », dans Sociology of Education, Vol. 49, No 2, p. 175-183. 